\title{
Validation of the Breast Cancer Awareness Scale Indonesian (BCAS-I) in Yogyakarta, Indonesia
}

\author{
Solikhah Solikhah ${ }^{1, *}$, Monthida Sangruangake ${ }^{2}$, Supannee Promthet ${ }^{3,4}$, and Cameron P. Hurst ${ }^{5}$ \\ ${ }^{1}$ Faculty of Public Health, Universitas Ahmad Dahlan, Indonesia \\ ${ }^{2}$ Faculty of Nursing, Ratchathani University, Udonthani, Thailand \\ ${ }^{3}$ Department of Epidemiology and Biostatistics, Faculty of Public Health, Khon Kaen University, Khon Kaen 40002, Thailand \\ ${ }^{4}$ ASEAN Cancer Epidemiology and Prevention Research Group, Khon Kaen University, Khon Kaen, Thailand \\ ${ }^{5}$ Biostatistics Center of Excellence, Faculty of Medicine, Chulalongkorn University, Bangkok 10330, Thailand
}

\begin{abstract}
Breast cancer is the commonest issue in public health. The screening method particularly clinically-breast examination is likely to drive the leading for early diagnosis of breast cancer, and breast cancer awareness is the key to encouraging in help seeking these treatments. Several breast cancer awareness instruments have been developed that the authors report as validated. However, instruments like those are highly contextualized to particular health care settings. The aim of this study was to develop the BCAS instrument among Indonesian women. Indonesian women aged 18 to 80 years were selected by stratified random sampling in five municipalities in Yogyakarta provinces by two locations (rural-urban) combinations were collected. Parallel analysis based on confirmatory factor analysis was conducted. The association of the BCAS-I subscales with participant characteristics was undertaken using proportional odds ordinal logistic regression. The questionnaires were completed by 200 Indonesian women (100\% response rate). The average age was $36(S D=12)$ and ranged from 18 to 74 years old. Most participants resided in rural areas (62\%), and 60\% didn't have health insurance. According to the parallel analysis, a five-factor solution was used. The factor solution was followed: knowledge of risk factors, knowledge of signs and symptoms, attitude to breast cancer prevention, a barrier of breast screening, and health behavior related to breast cancer awareness. And the alpha value of 0.78 indicates sufficiently high reliability to provide confidence interpreting the score. This study demonstrated that the BCAS-I tool is shown to valid and reliable in Yogyakarta women, Indonesia. Psychometric properties are needed to determine the construct, concurrent, criterion, and predictive validity of the BCAS-I scale.
\end{abstract}

Keywords: Breast Cancer Awareness Scale, Psychometric Properties, Indonesian Women.

\section{INTRODUCTION}

Globally, breast cancer is the commonest issue in public health. Breast cancer is second diagnosed in the globe. The incidence rate of breast cancer predicted a quarter million new cancer cases of overall cancer cases in worldwide. While the mortality rate of breast cancer is the fifth cause of death in the total of cancer mortality. ${ }^{1-3}$ In developing countries, breast cancer is the highest incidence. ${ }^{1}$ Breast cancer may be cured through changing lifestyle, including less physical activity, higher levels of obesity and alcohol consumption. ${ }^{2}$

Moreover, breast cancer death is rising in Asian women. ${ }^{4-6}$ Meanwhile, breast cancer incidence is the famous new cases in Indonesia in particular, ${ }^{7,8}$ and one of the problems in developing countries, such as Indonesia is that diagnosis of breast cancer occurs late presentation in breast cancer.

*Author to whom correspondence should be addressed.
Several campaigns to reduce the mortality rate of breast cancer have been trialed in developing countries, while difficulties in the deployment drive to the inefficiency of such programs, particularly in low and middle-income countries. ${ }^{9}$ Those due to no routine mammographic in their application in health care settings, ${ }^{10,11}$ Limited financial and other resources considered in those countries, awareness breast cancer is possible may reduced mortality rate in breast cancer. Thus early diagnosis in breast cancer can achieve through their empowering in these programs.

Socioeconomic of Indonesian women, belief, and culture led predictor variables with late presentation in breast cancer. ${ }^{12}$ Besides, lower in help seeking in a treatment of breast cancer seems to influence poor survival time of breast cancer. ${ }^{13-15}$ Early warning of breast cancer through breast cancer awareness is essential to reducing the mortality rate of breast cancer in particular. Furthermore, increasing awareness represents a feasible and costly effective method in countries with resource-limited 
health care, especially those that don't have ongoing organized population-based screening. ${ }^{15}$

The screening method particularly clinically-breast examination is likely to drive the leading for early diagnosis of breast cancer, and breast cancer awareness is the key to encouraging in help seeking in a treatment of breast cancer. Numerous studies into breast cancer awareness have been conducted in the West ${ }^{16-19}$ and breast cancer awareness instruments used for these studies have been developed especially for this context, and are unlikely to be valid in developing countries. In recent days, adequately validated instruments for breast cancer awareness seems conducted across cultures, or even outside the health care setting in which they were developed.

Several breast cancer awareness instruments have been developed that the authors report as validated. For example, breast$\mathrm{BCAM}^{17}$ was developed for women in the UK. However, instruments like those are highly contextualized to particular health care settings. Indeed, BCAM actually mentions the British mammographic screening program among its items, reducing its usefulness outside of the UK setting. The inadequacy of a Western developed breast cancer awareness tools for application to Asian populations is likely to lead to several problems. Beliefs and misconceptions are likely to be driven, at least in part, by cultural context. ${ }^{20-24}$ An instrument developed and validated on Western women may not be assumed to be valid in Asian populations.

The B-CAS (Breast Cancer Awareness Scale) tool was developed and validated for measuring breast cancer awareness in Thai women. ${ }^{25}$ Unlike many previously developed instruments for measuring breast cancer awareness, the B-CAS went through an appropriate and thorough validation. The aim of this study was developing the BCAS instrument among Indonesian women.

\section{MATERIALS AND METHOD}

\subsection{Participants}

Indonesian women aged 18 to 80 years were selected by stratified random sampling in five municipalities in Yogyakarta provinces by two location (rural-urban) combinations were collected. We felt these municipality-location combinations adequately represented the spectrum of cultures, religions, and socioeconomics in Yogyakarta women. Then, the questionnaire was administered in March to May 2016 to women with no history of breast cancer, who were neither pregnant nor breastfeeding, and was literate in the Indonesian language.

Measuring sample size for establishing psychometric properties is not clearly defined. However, a total of 100 participants has represented a sufficient sample size of evaluating explanatory factor analysis (EFA) ${ }^{26}$ All of the total 200 Yogyakarta women were obtained from all questionnaires distributed (response rate $=$ $100 \%$ ). Informed consent was obtained from all individual participants included in this study. The approving research was issued by the Ethics Committee of Khon Kaen University (HE582369), Thailand and a letter research permit from The Indonesian Ministry of Home Affairs (No. 440.02/1085/Polpum).

\subsection{Measurement}

The BCAS-I instrument divided into two sections. Firstly, the BCAS-I contained 14 questions as follows: age, province, region, educational level, marital status, monthly income, religion, current occupation, health insurance, family breast cancer history, family (any) cancer history, tobacco use, alcohol consumption, breastfeeding history. Secondly, the BCAS-I contained five domains include knowledge of risk factors, knowledge of signs and symptoms, attitude to breast cancer prevention, a barrier of breast screening, and health behavior related to breast cancer awareness. The two knowledge domains were weighed as yes/don't know/no. The domain of both attitudes to breast cancer prevention and barriers to breast screening were rated using a 5-point Likert scale from strongly disagree to agree strongly. The health behavior related to breast cancer awareness domain was measured using a 5-point frequency scale.

\subsection{Statistical Analysis}

Demographic data of the participants in this study were summarized using descriptive statistics. EFA and confirmatory factor analysis (CFA) was a method to confirm the construct validity of instrument model so as to seek a single or set of predefined constructs. $^{27,28}$ EFA was used to determine the number of latent factors (initially) or the pattern of relationships between the common factors and the indicators. CFA was used to test and modify the model that emerged from the EFA and specify the number of factors and the pattern of indicator-factor loadings. ${ }^{29}$ Parallel analysis based on Principal Components Analysis was used to determine the nature of the underlying factors. ${ }^{30}$ An Oblique (Promax) model was chosen after comparing principal axis factoring models with orthogonal (Varimax) and oblique rotations. The proposed measurement model is provided in Figure 1.

Cronbach's alpha coefficient examined the internal consistency of the BCAS-I instrument. Reliability coefficient over 0.70 was considered acceptable. ${ }^{31}$ Finally, the association of the BCAS-I subscales with participant characteristics was undertaken using proportional odds ordinal logistic regression. The data was coded in Epidata software version 3.1, and all analysis was performed using the $\mathrm{R}$ statistics package (R CRAN team version 2.3.0, 2015); the $\mathrm{R}$ libraries Lavaan ${ }^{32}$ and semPlot. ${ }^{33}$ A significance level of 0.05 will be used throughout all analysis.

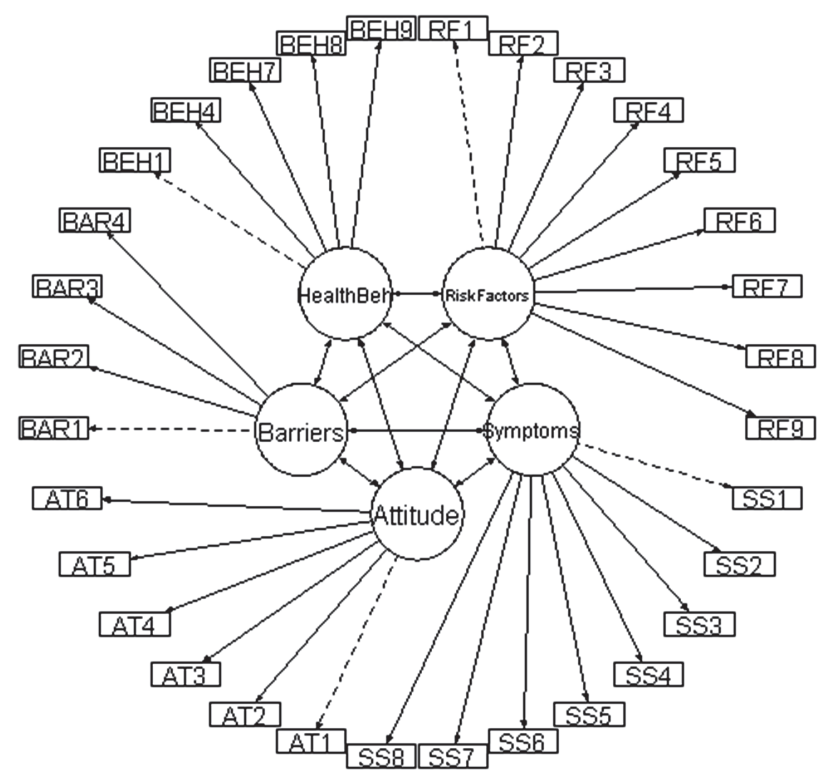

Fig. 1. Measurement model for breast cancer awareness scale Indonesian (BCAS-I). 


\section{RESULTS AND DISCUSSION}

\subsection{Sample Characteristics}

The questionnaires were completed by 200 Indonesian women $(100 \%$ response rate). The average age was $36(\mathrm{SD}=12)$ and ranged from 18 to 74 years old. Most participants resided in rural areas (62\%), and 60\% didn't have health insurance. The participant characteristics are shown in Table I.

Principal axis factoring with oblique rotations was then employed to explore the factor structure and the resulting loadings are given in Figure 2. And the coefficient in this pattern matrix was reported in Table II. The factor solution was following: (1) knowledge of risk factors (RF: 9 items), knowledge of signs and symptoms (SS: 8 items), attitude to breast cancer prevention (AT: 6 items), barrier of breast screening (BAR: 4 items),

Table I. Characteristics of breast cancer awareness in the confirmatory factor analysis.

\begin{tabular}{|c|c|c|}
\hline Items & Number & $\%$ \\
\hline \multicolumn{3}{|l|}{ Age } \\
\hline Early adulthood (18-34 y) & 99 & 49.3 \\
\hline Adulthood (35-59 y) & 84 & 41.8 \\
\hline Elderly (>60 y) & 7 & 3.5 \\
\hline \multicolumn{3}{|l|}{ District } \\
\hline Yogyakarta & 24 & 12 \\
\hline Bantul & 60 & 30 \\
\hline Sleman & 54 & 27 \\
\hline Kulonprogo & 23 & 11 \\
\hline Gunungkidul & 40 & 20 \\
\hline \multicolumn{3}{|l|}{ Locale } \\
\hline Rural & 50 & 25 \\
\hline Urban & 151 & 75 \\
\hline \multicolumn{3}{|l|}{ Education level } \\
\hline Primary school & 19 & 9.5 \\
\hline Junior high school & 17 & 8.5 \\
\hline Senior high school & 84 & 41.8 \\
\hline Bachelor degree & 74 & 36.8 \\
\hline Postgraduate degree & 7 & 3.5 \\
\hline \multicolumn{3}{|l|}{ Marital status } \\
\hline Single & 46 & 23 \\
\hline Married & 145 & 72 \\
\hline Widowed/separate/discovered & 10 & 5 \\
\hline \multicolumn{3}{|l|}{ Monthly income } \\
\hline$<2,000,000$ IDR & 155 & 77.1 \\
\hline$\geq 2,000,000$ to $6,000,000$ IDR & 46 & 22.9 \\
\hline \multicolumn{3}{|l|}{ Religion } \\
\hline Muslim & 92 & 95.5 \\
\hline Christians & 4 & 2 \\
\hline Others & 5 & 2.5 \\
\hline \multicolumn{3}{|l|}{ Occupation } \\
\hline No employment (include housewives) & 34 & 17 \\
\hline Farmer & 23 & 11 \\
\hline Trader & 30 & 15 \\
\hline Laborer & 49 & 24 \\
\hline Government/official/enterprise/business & 36 & 18 \\
\hline Student & 29 & 14 \\
\hline Others & & \\
\hline \multicolumn{3}{|l|}{ Health insurance } \\
\hline Yes & 123 & 61 \\
\hline No & 78 & 39 \\
\hline \multicolumn{3}{|l|}{ Smoking history } \\
\hline Yes & 2 & 1 \\
\hline No & 199 & 99 \\
\hline \multicolumn{3}{|l|}{ Alcohol history } \\
\hline Yes & 200 & 99.5 \\
\hline No & 1 & 0.5 \\
\hline
\end{tabular}

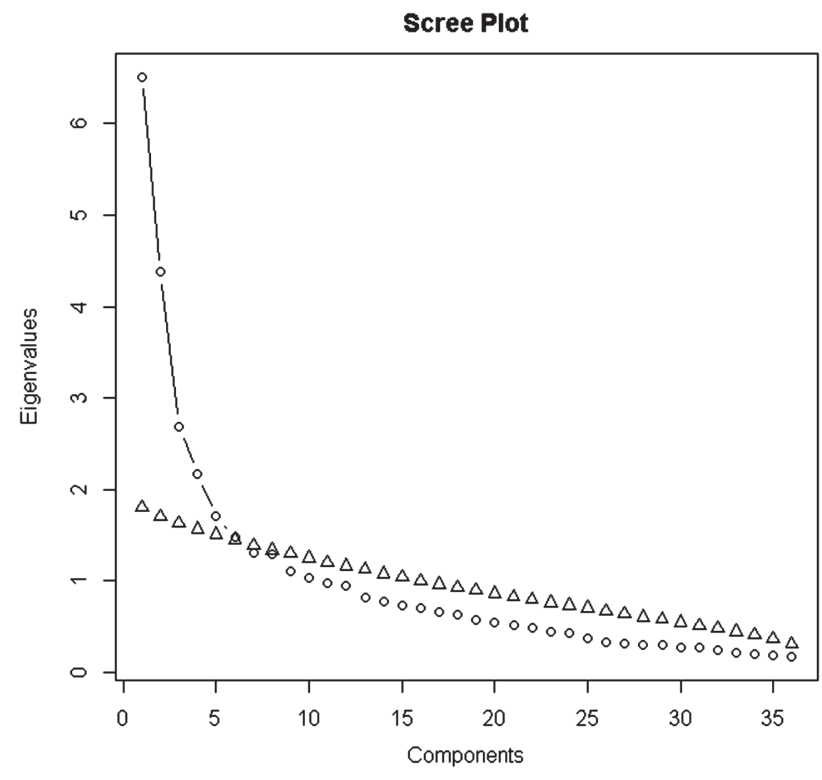

Fig. 2. Parallel analysis derived from a confirmatory factor analysis.

and health behavior related to breast cancer awareness (BEH: 6 items).

\subsection{Reliability}

The reliability of the BCAS-I was evaluated using Cronbach's alpha demonstrated that a good level of internal consistency with the alpha value of 0.78 indicates sufficiently high reliability to provide confidence interpreting the score.

\section{DISCUSSION}

We successfully developed the first instrument of breast cancer awareness Indonesian scale (BCAS-I) based on Yogyakarta, Indonesian women who were adapted to cultural contexts/health care settings in Indonesia. According to the factor analysis, we produced factors considered breast cancer awareness scale as follows: (1) knowledge of risk factors (RF: 9 items), knowledge of signs and symptoms (SS: 8 items), attitude to breast cancer prevention (AT: 6 items), barrier of breast screening (BAR: 4 items), and health behavior related to breast cancer awareness (BEH: 6 items). The results of this study indicated the factor solution for the BCAS-I scale is promising to use in Indonesian culture context. The internal consistency demonstrated adequate reliability for the instrument measuring four subscales, as all subscales were higher than 0.70. Also, all items of the BCAS-I structure were strongly well with the hypothesis in their construct. Moreover, we regard to the construct validity, and all participants characteristics indicated that they obtained strongly association on each subscale. We also found the BCAS-I has reached validation strongly to lend particular importance to our BCAS-I-based study because it constitutes the first step towards achieving better understanding women's awareness knowledge about breast cancer and symptoms of the disease. It can encourage them to perform better and timelier breast self-exams among Indonesian women. The BCAS-I can likely lead evaluating breast cancer awareness to achieve prevention strategies through public education to reduce the known risk factor of breast cancer. ${ }^{34-37}$ 
Table II. Standardized loading factors for explanatory factor analysis in the BCAS-I.

\begin{tabular}{|c|c|c|c|c|c|c|c|}
\hline \multirow[b]{2}{*}{ Domains } & \multirow{2}{*}{$\begin{array}{l}\text { Standardized loading } \\
\text { factors }\end{array}$} & \multicolumn{5}{|c|}{ Factor loading } & \multirow[b]{2}{*}{ h2 } \\
\hline & & 1 & 2 & 3 & 4 & 5 & \\
\hline \multicolumn{8}{|l|}{ Knowledge of risk factors ( 9 items) } \\
\hline RF1 Family history of breast cancer & 0.500 & & 0.26 & & & & 0.278 \\
\hline RF2 Use of birth control pills & 0.516 & & 0.38 & & & & 0.240 \\
\hline RF3 Having undergone hormone replacement therapy & 0.737 & & 0.40 & & & & 0.454 \\
\hline RF4 Beginning your menses before the age of 12 & 0.589 & & 0.64 & & & & 0.486 \\
\hline RF5 Menopause after the age of 55 & 0.541 & & 0.78 & & & & 0.575 \\
\hline RF6 Infertility & 0.664 & & 0.71 & & & & 0.579 \\
\hline RF7 Giving birth after the age of 30 & 0.601 & & 0.69 & & & & 0.501 \\
\hline RF8 Eating fatty foods & 0.577 & & 0.35 & & & & 0.363 \\
\hline RF9 Obesity & 0.561 & & 0.39 & & & & 0.368 \\
\hline \multicolumn{8}{|l|}{ Knowledge signs and symptoms (8 items) } \\
\hline SS1 Bleeding or liquid discharge from the nipple & 0.640 & & & 0.60 & & & 0.433 \\
\hline SS2 Swelling in the breast or armpit area. & 0.801 & & & 0.79 & & & 0.757 \\
\hline SS3 Changes in the shape, size or color around the breast or nipple & 0.668 & & & 0.50 & & & 0.472 \\
\hline SS4 Pain in the breast or armpit area & 0.719 & & & 0.71 & & & 0.589 \\
\hline SS5 Sensation of the nipple being pulled from the inside & 0.629 & & & 0.46 & & & 0.345 \\
\hline SS6 A lump or thickness of skin beneath the armpit & 0.716 & & & 0.61 & & & 0.541 \\
\hline SS7 Puckering or dimpling/scaling around the breast & 0.753 & & & 0.53 & & & 0.482 \\
\hline SS8 A lump or thickness of skin in the breast area & 0.743 & & & 0.66 & & & 0.605 \\
\hline \multicolumn{8}{|l|}{ Attitude to breast cancer prevention ( 6 items) } \\
\hline $\begin{array}{l}\text { AT1 Breast cancer can be avoided by decreasing the risk } \\
\text { factors of breast cancer }\end{array}$ & 0.688 & 0.66 & & & & & 0.555 \\
\hline AT2 Breast cancer can be cured if it is detected in its first stage & 0.820 & 0.75 & & & & & 0.650 \\
\hline $\begin{array}{l}\text { AT3 Breast cancer can be detected in its first stage by having routine } \\
\text { check-ups with a doctor/health specialist }\end{array}$ & 0.813 & 0.71 & & & & & 0.567 \\
\hline AT4 Routine mammogram tests can detect breast cancer in its first stage. & 0.810 & 0.69 & & & & & 0.540 \\
\hline AT5 Exercise can reduce the risk of breast cancer & 0.518 & 0.55 & & & & & 0.413 \\
\hline AT6 Reducing fatty foods can decrease the risk of breast cancer & 0.397 & 0.48 & & & & & 0.370 \\
\hline \multicolumn{8}{|l|}{ Barrier of breast screening (4 items) } \\
\hline BAR1 I don't feel comfortable going to the doctor for a breast screening & 0.690 & & & & 0.59 & & 0.488 \\
\hline BAR2 Breast check-ups involve too much time waiting in line & 0.701 & & & & 0.62 & & 0.483 \\
\hline BAR3 I am busy and don't have time to go the doctor for a breast check-up & 0.649 & & & & 0.65 & & 0.463 \\
\hline BAR4 I don't know how to check a breast self-examination & 0.734 & & & & 0.55 & & 0.491 \\
\hline \multicolumn{8}{|l|}{ Health behavior related to breast cancer awareness (7 items) } \\
\hline BEH1 How often do you eat fried food? & 0.057 & & & & & 0.28 & 0.111 \\
\hline BEH4 How often do you eat fresh vegetables? & -0.063 & & & & & 0.27 & 0.082 \\
\hline BEH7 How often did you perform breast self examination by yourself & -2.419 & & & & & 0.36 & 0.406 \\
\hline BEH8 How often do you get breast check-ups at health clinics? & -0.093 & & & & & 0.62 & 0.422 \\
\hline BEH9 How often do you get mammogram tests? & -0.028 & & & & & 0.51 & 0.305 \\
\hline
\end{tabular}

Notes: *** Strongly loading items; +: Item constrained.

In present results in this study showed reaching an Indonesian scale of breast cancer awareness with 33 items were included in a 5-domains model for the CFA of the BCAS-I. According to the dimensions available in previous studies and the dimensions present from this study, a conceptual model was created to show the relationship between the factors underlying breast cancer awareness (Fig. 3). We validated the BCAS-I, achieving an ideal fit without two the original items of health behavior associated with breast cancer awareness 2 (How many days per week do you eat food or dessert with coconut?) and 3 (How often do you eat beef, chicken, or duck with the fat or skin?). Two items of health behavior (e.g., BEH 2 and BEH 3) were removed due to it did not load significantly on its corresponding structure in the BCAS-I. Nonetheless, the high internal consistency reliability was reached in our instrument. A good model fit of the BCAS-I was in line with previous work testing the psychometric properties of English, and the Thai version of these scales. ${ }^{16,17,25,38}$ Why is ours better than others (e.g., the BCAM) ${ }^{17}$ was developed specially for the UK health system and actually refers to specific programs (e.g., mammography screening) offered this is health setting. The BCAM is unlikely to be appropriate anywhere outside of the UK. B-CAS, on the other hand, could be only adjusted for different cut point; indeed it was originally developed for Thai women. The BCAS-I, this was likely adapted to the cultural context and a particular health setting in Indonesia.

The limitation of this study was acknowledgment. According to cross-sectional in this study, some bias was identified, such as most of Indonesian is well known as rich culture and Muslim. In order taking one province particularly, Yogyakarta may not represent the several cultures and ethnic of Indonesian. Also, another limitation is the most of the women was poor educated which was inadequate knowledge of breast cancer might raise concerns about selection bias.

Strengths of this study should be noted. This study is the first develop in Indonesian women particularly in Yogyakarta. Although only women were included in this study, they demonstrated a relatively wide range of age (from 18 to 80 ) years) and were distributed across various education levels.

\section{CONCLUSION}

Finally, we concluded the results of this study demonstrated that the BCAS-I tool is shown to valid and reliable in Yogyakarta 
women, Indonesia. In the future, psychometric is needed to determine the construct, concurrent, criterion, and predictive validity of the BCAS-I scale.

\section{Conflict of Interest}

The authors declare that there is no conflict of interests regarding the publication of this paper.

\section{References and Notes}

1. J. Ferlay, I. Soerjomataram, R. Dikshit, et al., Int. J. Cancer 136, E359 (2015).

2. A. Jemal, F. Bray, M. M. Center, J. Ferlay, E. Ward, and D. Forman, CA Cancer J. Clin. 61, 69 (2011).

3. A. Jemal, M. M. Center, C. DeSantis, and E. M. Ward, Cancer Epidemiol. Biomarkers Prev. 19, 1893 (2010)

4. M. A. Moore, Y. Ariyaratne, F. Badar, et al., Asian Pac. J. Cancer Prev. APJCP 11 (Suppl. 2), 49 (2010).

5. E. I. Salim, M. A. Moore, A. Bener, O. S. Habib, I. A.-B. Seif-Eldin, and T. Sobue, Asian Pac. J. Cancer Prev. 11 (Suppl. 2), 33 (2010).

6. M. A. Moore, S. Eser, N. Igisinov, et al., Asian Pac. J. Cancer Prev. 11(Suppl. 2), 17 (2010)

7. M. Wahidin, R. Noviani, S. Hermawan, V. Andriani, A. Ardian, and H. Djarir, Asian Pac. J. Cancer Prev. 13, 1709 (2012)

8. C. H. Ng, N. B. Pathy, N. A. Taib, et al., Asian Pac. J. Cancer Prev. APJCP 12, 2943 (2011).

9. M. S. Hossain, S. Ferdous, and H. E. Karim-Kos, Cancer Epidemiol. 38, 465 (2014)

10. S. S. Coughlin and D. U. Ekwueme, Cancer Epidemiol. 33, 315 (2009).

11. N. S. El Saghir, C. A. Adebamowo, B. O. Anderson, et al., The Breast 20 (Suppl. 2), S3 (2011)

12. M. Toure, E. Nguessan, A. T. Bambara, Y. K. K. Kouassi, J. M. L. Dia, and I. Adoubi, Gynecol. Obstet. Fertil. 41, 696 (2013).

13. A. Bish, A. Ramirez, C. Burgess, and M. Hunter, J. Psychosom. Res. 58, 321 (2005).

14. L.-Y. Liu, F. Wang, L.-X. Yu, et al., BMC Public Health 14, 1 (2014).

15. D. R. Youlden, S. M. Cramb, C. H. Yip, and P. D. Baade, Cancer Biol. Med 11, 101 (2014)
16. S. Stubbings, K. Robb, J. Waller, et al., Br. J. Cancer 101 (Suppl. 2), S13 (2009).

17. L. Linsell, L. J. L. Forbes, C. Burgess, M. Kapari, A. Thurnham, and A. J. Ramirez, Eur. J. Cancer 46, 1374 (2010)

18. E. Özalp, E. H. Karslığlu, Ö. Aydemir, et al., Sex Disabil. 33, 253 (2014)

19. D. Pud, J. Pain Symptom Manage. 49, 790 (2015).

20. A.-A. Mohammed, A.-A. Huda, and A.-M. Mansour, Breast J. 15, 615 (2009).

21. M. Ahmadian and A. A. Samah, Life Sci. J. 9, 585 (2012).

22. A. E. Simon, L. J. L. Forbes, D. Boniface, et al., BMJ Open 2,1 (2012).

23. P. Gonzalez, J.-W. Lim, M. Wang-Letzkus, et al., West J. Nurs. Res. 37, 1081 (2015).

24. B. Norsa'adah, M. A. Rahmah, K. G. Rampal, and A. Knight, Asian Pac. J. Cancer Prev. 13, 3723 (2012).

25. N. Rakkapao, S. Promthet, M. A. Moore, and C. P. Hurst, Asian Pac. J. Cancer Prev. APJCP 17, 851 (2016)

26. E. Anthoine, L. Moret, A. Regnault, V. Sébille, and J.-B. Hardouin, Health Qual. Life Outcomes 12 (2014), DOI: 10.1186/s12955-014-0176-2.

27. W. Revelle, An Overview of the Psych Package, Department of Psychology Northwestern University (2015), https://cran.r-project.org/web/packages/ psych/vignettes/overview.pdf.

28. J. H. Steiger, Confirmatory Factor Analysis with R. Spring (2013), http:// www.statpower.net/Content/312/Handout/Confirmatory\%20Factor\%20Analysis \%20with\%20R.pdf, Accessed September, 2015.

29. A. B. Costello and J. W. Osborne, Best Practices in Exploratory Factor Analysis: Four Recommendations for Getting the Most from Your Analysis (2005), Vol. 10, pp. 1-9.

30. S. P. Reise, N. G. Waller, and A. L. Comrey, Psychol. Assess 12, 287 (2000).

31. K. Sijtsma, Psychometrika 74, 107 (2009).

32. R. Y. Lavaan, J. Stat Softw. 48, 1 (2012).

33. S. Epskamp, Path diagrams and visual analysis of various SEM packages' output, February (2015)

34. A.-A. Lertkhachonsuk, C. H. Yip, T. Khuhaprema, et al., Lancet Oncol. 14, e497 (2013)

35. H. Ma, L. Bernstein, M. C. Pike, and G. Ursin, Breast Cancer Res. BCR 8, R43 (2006).

36. A. McTiernan, C. Kooperberg, E. White, et al., JAMA 290, 1331 (2003)

37. L. M. Morimoto, E. White, Z. Chen, et al., Cancer Causes Control CCC 13, 741 (2002).

38. S. Solikhah, S. Promthet, N. Rakkapao, and C. P. Hurst, Asian Pac. J. Cancer Prev. 18, 515 (2017)

Received: 2 July 2017. Accepted: 22 July 2017. 